\title{
Cecal perforation with an ascending colon cancer caused by upper gastrointestinal endoscopy
}

\author{
Hiroyuki Miyatani' \\ Yukio Yoshida' \\ Hirokazu Kiyozaki² \\ 'Department of Gastroenterology, \\ Jichi Medical University, Saitama \\ Medical Center, Saitama, Japan; \\ ${ }^{2}$ Department of Surgery, Jichi Medical \\ University, Saitama Medical Center, \\ Saitama, Japan
}

\begin{abstract}
Colonic perforation caused by upper gastrointestinal (GI) endoscopy is extremely rare. A 69-year-old woman was referred to our hospital because of abdominal fullness. Colonoscopy could be performed only up to the hepatic flexure due to an elongated colon and residual stools. Because her symptoms improved, upper GI endoscopy was performed 11 days later. The patient developed severe abdominal pain two hours after the examination. Abdominal X-ray and computed tomography showed massive free air. Immediate laparotomy was performed for the intestinal perforation. After removal of stool, a perforation site was detected in the cecum with an invasive ascending colon cancer. Therefore, a right hemicolectomy, ileostomy, and transverse colostomy were performed. Although she developed postoperative septicemia, the patient was discharged 38 days after admission. Seven months postoperatively, the patient died of lung, liver, and brain metastases. Even in cases with a lesion that is not completely obstructed, it is important to note that air insufflations during upper GI endoscopy can perforate the intestinal wall in patients with advanced colon cancer.
\end{abstract}

Keywords: colonic perforation, colon cancer, upper gastrointestinal endoscopy, fecal peritonitis

\section{Introduction}

Colorectal cancer with perforation is a rare, severe condition. Colonic preparation for colonoscopy or barium enema rarely causes colonic perforation in cases of colonic obstruction and stenosis. ${ }^{1}$ However, there have been few reports of colonic perforation caused by air insufflations during upper gastrointestinal (GI) endoscopy. We report a cecal perforation in a patient with an ascending colon cancer after upper GI endoscopy.

\section{Case report}

A 69-year-old woman was referred because of abdominal fullness. She complained of intermittent nausea and had vomited for two months. She had no weight loss, hematochezia, and melena. There were no temporal relationship between meals and her symptom. She had normal bowel movements and no abdominal pain. On physical examination, the patient appeared to be in no acute distress. Her abdomen was slightly distended without tenderness or a mass. An abdominal X-ray showed a large amount of stool in the colon (Figure 1). The serum carcinoembryonic antigen (CEA) $(7.3 \mathrm{ng} / \mathrm{ml})$ was slightly elevated. Other laboratory data were normal. Colonoscopy was performed after a retention enema. Unfortunately endoscopic examination could be performed only up to the hepatic flexure due to an elongated colon and residual stools. No abnormal lesion was found. Because of her symptomatic improvement and low probability of colonic obstruction, screening upper GI endoscopy was performed to find the cause of vomiting 11 days later. The upper GI endoscopy revealed mild gastropathy and a small benign-appearing polyp without abnormal findings in the duodenum. Endoscopic examination was routinely finished without biopsy. 


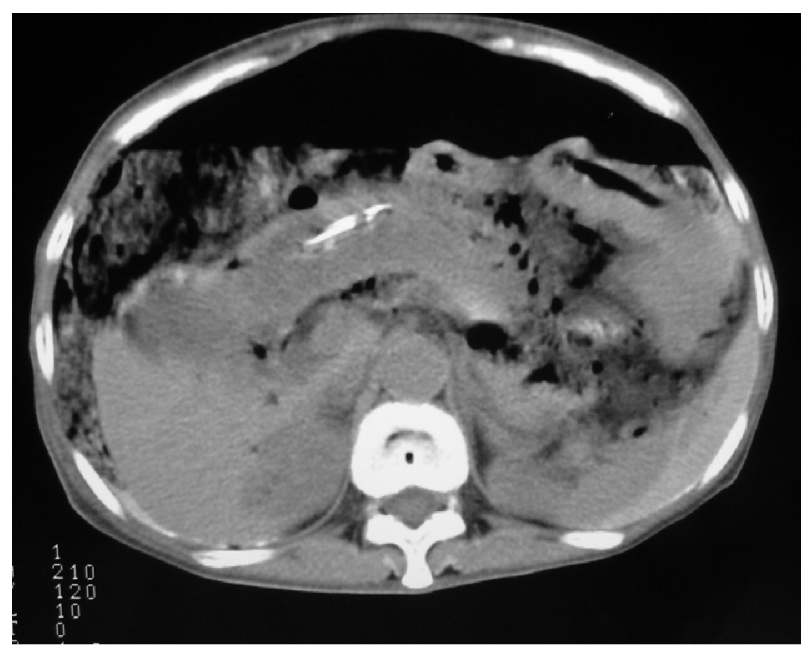

Figure I Abdominal computed tomography showed massive free air and stools in the abdominal cavity.

The patient developed severe abdominal pain two hours after the examination and returned. Her systolic blood pressure was $78 \mathrm{mmHg}$ by palpation, her abdomen was diffusely distended, and bowel sounds were absent. Abdominal X-ray and computed tomography (CT) showed massive free air. Immediate laparotomy was performed for the intestinal perforation. There was a massive amount of stool in the abdominal cavity. After removal of the stool, a perforation site was detected in the cecum with an invasive near-circumferential ascending colon cancer (Figure 2). The perforation site was separated from the tumor. There was no evidence of metastasis except for direct invasion to the duodenal serosa which could be easily peeled. Therefore, a right hemicolectomy, ileostomy, and transverse colostomy were performed. Although she developed postoperative septicemia, the patient was discharged 38 days after admission. Seven months postoperatively, the patient died of lung, liver, and brain metastases.

\section{Discussion}

Large bowel perforation is a rare and severe complication of colorectal cancer. The prognosis is very poor, with a postoperative mortality rate ranging from $6.5 \%$ to $30 \% .^{2,3}$ Furthermore, the postoperative complication rate is high after a free perforation. ${ }^{4}$ In general, there are two mechanisms of perforation: 1) direct perforation at the tumor site due to tumor necrosis or adhesion to other organs, and 2) proximal colon blow-out due to necrosis of the colonic wall due to excess preload. When colonic perforation occurs in colon cancer, it is either spontaneous or due to an obvious trigger, including iatrogenic causes. Because of the risk of intestinal perforation, colonic preparation for colonoscopy and barium enema should be considered contraindicated in patients with intestinal obstruction. However, there have been only a few previous reports of free perforations caused by upper GI

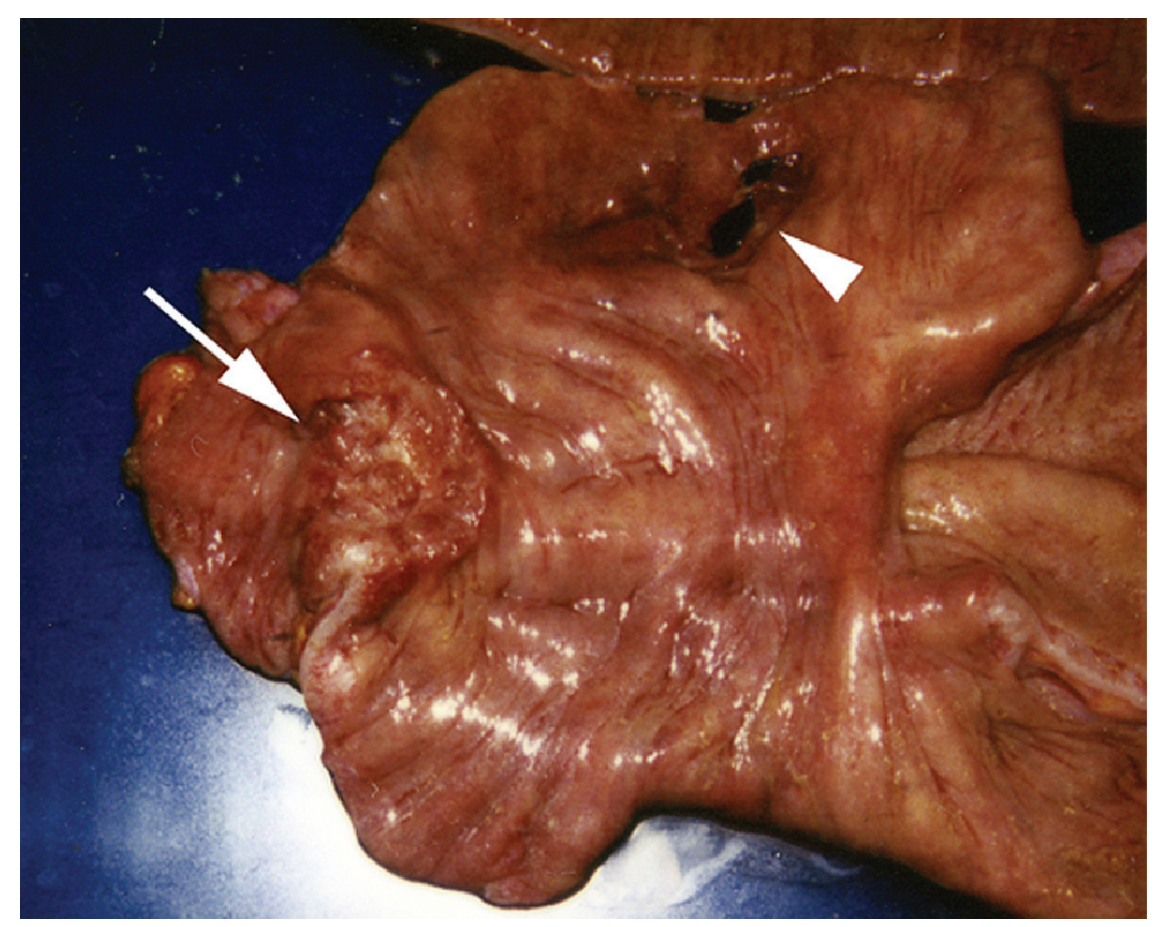

Figure 2 Gross appearance of the resected colon and small intestine. Advanced ascending colon cancer (arrow) and cecal perforation site (arrow head) were found. 
endoscopy. ${ }^{5,6}$ In the present case, one can assume that air insufflations during upper GI endoscopy caused free perforation on the oral site of the stenosis caused by the ascending colon cancer. Despite the usual screening endoscopic procedure, it is conceivable that the air compressed and ruptured the vulnerable site of the intestinal wall, which then became necrotic due to chronic compression and a local circulatory disturbance. CT scan may be effective for detecting colonic tumor in patients complaining of abdominal fullness, as in the present case. ${ }^{7,8}$ However, urgent examination was considered unnecessary in the present case due to the patient's symptomatic improvement.

Colon cancer should not be assumed not to be present unless the colonoscopy is truly complete. We should have performed total colonoscopy at a later date after standard bowel preparation so as not to miss a proximal lesion. However, bowel preparation might cause colonic perforation in this case because of the stenosis of the colon.

Even in cases with a lesion that is not completely obstructed, it is important to note that air insufflations during upper GI endoscopy can perforate the intestinal wall in patients with advanced colon cancer.

\section{Disclosure}

The authors report no conflicts of interest in this work.

\section{References}

1. Galloway D, Burns HJ, Moffat LE, MacPherson SG. Faecal peritonitis after laxative preparation for barium enema. $\mathrm{Br}$ Med J (Clin Res Ed). 1982;284(6314):472.

2. Bielecki K, Kaminski P, Klukowski M. Large bowel perforation: morbidity and mortality. Tech Coloproctol. 2002;6(3):177-182.

3. Kriwanek S, Armbruster C, Dittrich K, et al. Perforated colorectal cancer. Dis Colon Rectum. 1996;39(12):1409-1414.

4. Welch JP, Donaldson GA. Perforative carcinoma of colon and rectum. Ann Surg. 1974;180(5):734-740.

5. Weiner BC. Complications of routine diagnostic upper endoscopy. Gastrointest Endosc. 1987;33(1):53.

6. Rex DK, Hawes RH, Goulet RJ. Cecal perforation after upper endoscopy and pyloric dilation. Gastrointest Endosc. 1988;34(6):490-491.

7. Xiong L, Chintapalli KN, Dodd GD, 3rd, et al. Frequency and CT patterns of bowel wall thickening proximal to cancer of the colon. AJR Am J Roentgenol. 2004;182(4):905-909.

8. Horton KM, Abrams RA, Fishman EK. Spiral CT of colon cancer: imaging features and role in management. Radiographics. 2000;20(2):419-430. 
